\title{
One-pot Synthesis of Reactive Base Metal Nanoparticles in Multifunctional Pyridine
}

\author{
Alexander Egeberg, ${ }^{\dagger}$ Tim P. Seifert,${ }^{\dagger}$ Peter W. Roesky,${ }^{\dagger}$ Dagmar Gerthsen,${ }^{\dagger}$ and Claus \\ Feldmann ${ }^{\dagger *}$
}

†Institute of Inorganic Chemistry, Karlsruhe Institute of Technology (KIT), Engesserstraße 15, 76131 Karlsruhe, Germany, claus.feldmann@kit.edu

Laboratory for Electron Microscopy, Karlsruhe Institute of Technology (KIT), Engesserstrasse 7, 76131 Karlsruhe, Germany. E-mail: dagmar.gerthsen@kit.edu

\section{- Supporting Information -}

\section{Contents}

1. Analytical Equipment

2. Surface Conditioning of the Metal Nanoparticles

3. Reaction Mechanism

4. $\mathrm{Zn}^{0}$ Nanoparticles (One-pot Approach)

5. Elemental Analysis 


\section{Analytical Equipment}

Transmission electron microscopy (TEM). TEM and high-resolution transmission electron microscopy (HR)TEM were conducted with a FEI Osiris microscope operating at $200 \mathrm{kV}$. TEM samples of the metal nanoparticles were prepared by evaporating pyridine suspensions on amorphous carbon (Lacey-)film suspended on copper grids. The deposition of the metal nanoparticles on the carbon (Lacey-)film copper grids was performed under argon atmosphere in a glovebox. Subsequently after preparation the $\mathrm{Sn}^{0}$ and $\mathrm{Zn}^{0}$ nanoparticles were dried under vacuum for at least 3 days to remove adhered volatiles. $\mathrm{V}^{0}$ and $\mathrm{Mn}^{0}$ nanoparticles were slowly heated to $130{ }^{\circ} \mathrm{C}$ in vacuum. Thereafter, the all grids were transferred with a suitable vacuum/inert gas transfer module into the transmission electron microscope without any contact to air. Average particle diameters were calculated by statistical evaluation of $>100$ particles (Image J $1.48 \mathrm{v}$ ).

$X$-ray powder diffraction (XRD). XRD was performed with a STOE STADI-MP diffractometer operating with Ge-monochromatized $\mathrm{Cu}-\mathrm{K}_{\alpha}$-radiation $(\lambda=1.54178 \AA)$. A background correction was applied to fit the non-specific scattering (Win-XPOW, 1.2v). Calculation of XRD powder patterns was performed via DIAMOND (Crystal Impact, Visuelles Informationssystem für Kristallstrukturen, Version 3.0d, Bonn, Germany, 2005) using CCDC-No. 1111461 as a reference. ${ }^{\mathrm{S} 1}$

$\mathrm{Zn}^{0}, \mathrm{Sn}^{0}, \mathrm{~V}^{0}$, and $\mathrm{Mn}^{0}$ nanoparticles were diluted and pestled with dried, amorphous silica spheres $(9-13 \mu \mathrm{m}$, Sigma-Aldrich) in a glove box, thereafter filled into glass capillaries $(0.3 \mathrm{~mm}$ in diameter), and then sealed under argon. Sample preparation in glass capillaries was required to perform the XRD analysis under inert conditions and to avoid any contact of the reactive base metal powders with oxygen and moisture.

Pestling and dilution of the base metal powders with amorphous silica spheres were necessary to fill the sticky metal nanoparticles in glass capillaries $(0.3 \mathrm{~mm}$ in diameter $)$ and to reduce the X-ray absorption of the metal nanoparticles. Pestling and dilution with amorphous silica spheres, however, caused a merging of the low melting $\mathrm{Zn}^{0}$ and $\mathrm{Sn}^{0}$ metal nanoparticles. Therefore, the as-treated $\mathrm{Zn}^{0}$ and $\mathrm{Sn}^{0}$ samples exhibit very narrow, bulk-like Bragg peaks (see main text: Figures 2d,11a; cf. Figure S6). The high-melting metals $\mathrm{V}^{0}$ and $\mathrm{Mn}^{0}$ did not show any merging when pestling with amorphous silica spheres. Due to the low scattering power of these small-sized metal nanoparticles (diameter $\leq 10 \mathrm{~nm}$ ), considerable Bragg peaks were only observed after sintering $\left(1000{ }^{\circ} \mathrm{C}, 10 \mathrm{~min}\right)$. Although, of course, no nanoparticles were available after sintering, the absence of any impurity phases (e.g., metal oxides, metal 
hydroxides, metal carbonates) nevertheless clearly validates the purity of the as-prepared $\mathrm{V}^{0}$ and $\mathrm{Mn}^{0}$ nanoparticles (see main text: Figures 11 b,c).

Elemental analysis (EA). $\mathrm{C} / \mathrm{H} / \mathrm{N}$ analysis was performed with an Elementar Vario Microcube device (Elementar, Hanau, Germany) at a temperature of about $1100^{\circ} \mathrm{C}$.

Mass spectrometry (MS). MS was conducted using a Q Exactive (Orbitrap) mass spectrometer (ThermoFisher) equipped with a heated electrospray ionization (HESI-II) probe. Measurements were conducted in absolute THF with $\mathrm{Na}(\mathrm{OTf})$.

Nuclear magnetic resonance (NMR) spectroscopy. NMR spectra of solutions (after centrifugation of the metal nanoparticles) were recorded on a Bruker Avance II instrument operating at a ${ }^{1} \mathrm{H}$-Larmor frequencies of $300 \mathrm{MHz}$. Chemical shifts are given in ppm.

Fourier-transformed infrared (FT-IR) spectroscopy. FT-IR spectra were recorded on a Bruker Vertex 70 FT-IR spectrometer in the area of 4000 to $400 \mathrm{~cm}^{-1}$ with a resolution of $4 \mathrm{~cm}^{-1}$. For transmission measurements, $1 \mathrm{mg}$ of the metal nanoparticles was ground using a mortar and pestled with $300 \mathrm{mg}$ of dried $\mathrm{KBr}$ and pressed to a pellet.

Centrifugation. All centrifugation of metal nanoparticles was performed with a superspeed refrigerated centrifuge Sigma 3-30KS. To guarantee inert conditions (argon), the centrifuge was placed in a glove box. Tubes with a volume of $30 \mathrm{~mL}$ were used and allow a maximum speed of $30.000 \mathrm{rpm}$ relating to about $65.000 \times \mathrm{g}$.

\section{Surface Conditioning of the Metal Nanoparticles}

The as-prepared $\mathrm{Zn}^{0}$ nanoparticles were used to exemplarily examine the surface conditioning. After successful synthesis of the $\mathrm{Zn}^{0}$ nanoparticles, pyridine and 2,2'-bipyridine are available in the suspension and can potentially serve as surface-stabilization agents. To elucidate the surface functionalization, FT-IR was performed (Figure S1). By comparison to pure pyridine and pure 2,2'-bipyridine, the spectra of the as-prepared $\mathrm{Zn}^{0}$ nanoparticles are in good agreement with pyridine as a reference (especially in the fingerprint area $1500-600 \mathrm{~cm}^{-}$ ${ }^{1}$ ), whereas certain differences are visible in regard of 2,2'-bipyridine (Figure S1). Thus, pyridine can be considered as the relevant surface functionalization of the as-prepared metal 
nanoparticles. Taking the great molar excess of pyridine as the solvent into account, in fact, this finding is not a surprise.

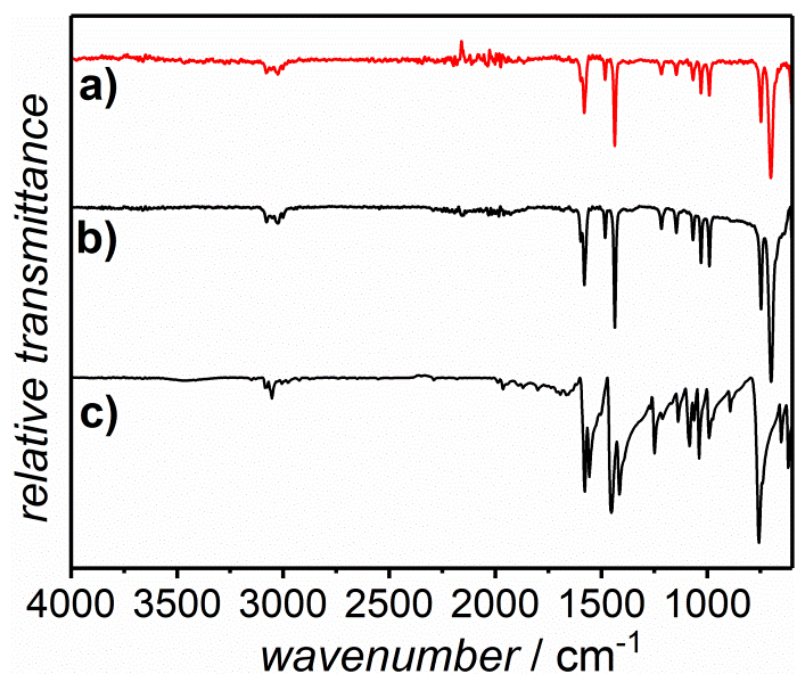

Figure S1. FT-IR spectra of: a) As-prepared $\mathrm{Zn}^{0}$ nanoparticles; b) Pure pyridine as a reference; c) Pure 2,2'-bipyridine as a reference.+

\section{Reaction Mechanism}

The colorless solid that was instantaneously formed in the first step (addition of $\mathrm{ZnCl}_{2}$ and $\mathrm{KNH}_{2}$ of the two-step liquid-ammonia approach was examined by XRD and clearly revealed the formation of $\mathrm{KCl}$ (Figure $\mathrm{S} 2$; see main text: Figure $1 b$ ). $\mathrm{Zn}\left(\mathrm{NH}_{2}\right)_{2}$ as intermediate compound remained amorphous and is therefore not observed on XRD patterns.

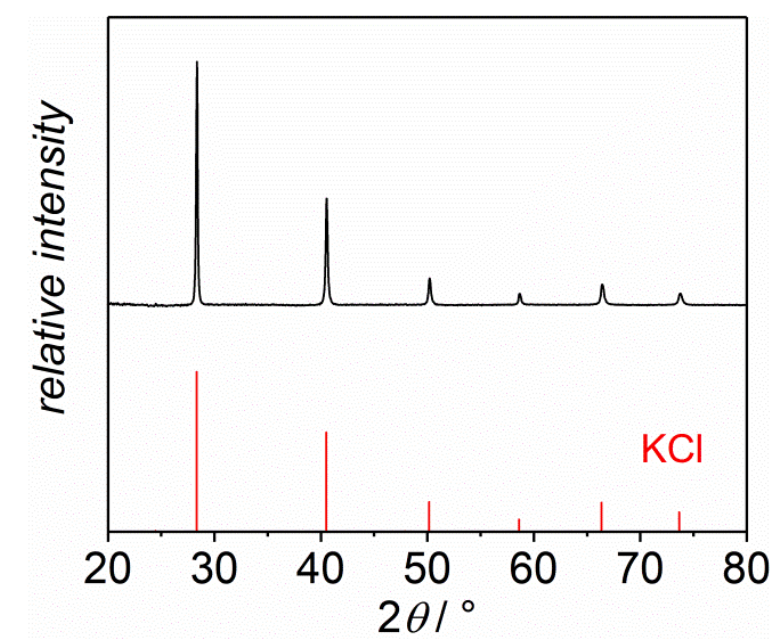

Figure S2. XRD pattern of $\mathrm{KCl}$ as by-product of the reaction of $\mathrm{ZnCl}_{2}$ and $\mathrm{KNH}_{2}$ (reference KCl ICDD-No. 01-074-9685). 
If the as-prepared $\mathrm{Zn}^{0}$ nanoparticles were separated by centrifugation and if all pyridine was distilled from supernatant, a colorless, crystalline powder remained (Figure S3). All Bragg peaks can be attributed to 2,2'-bipyridine (CCDC-No. 1111461). ${ }^{\mathrm{S} 1}$ In addition to MS and NMR (see main text: Figures 4,5), this finding as well validates the formation of 2,2'bipyridine.
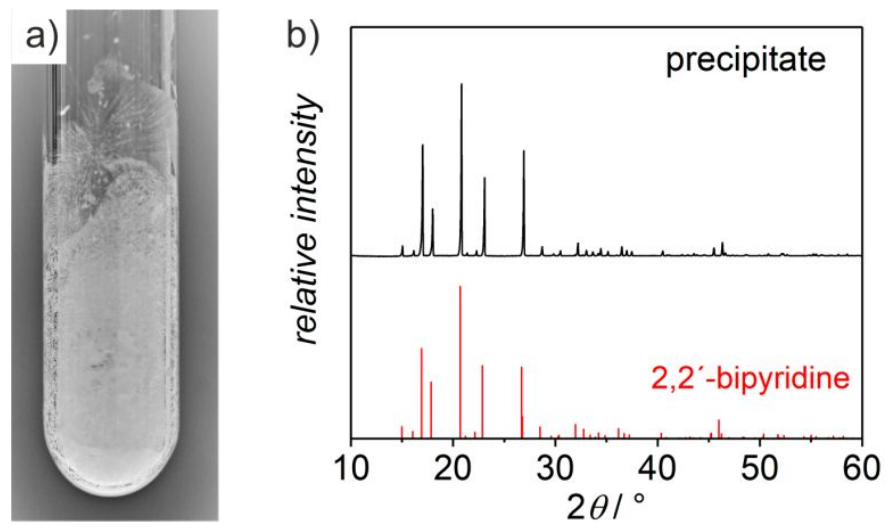

Figure S3. a) Photo of colorless residue obtained after centrifugation of the $\mathrm{Zn} 0$ nanoparticles and vacuum distillation of pyridine; b) XRD pattern of the colorless residue and reference pattern of 2,2'-bipyridine (CCDC-No. 1111461). ${ }^{\mathrm{S} 1}$

In order to validate the proposed mechanism of the pyridine-mediated reduction of base metals, we recognized slight overpressure of the autoclave subsequent to the reaction. In addition to the evidence of 2,2'-bipyridine as a product (Figure S3; see main text: Figures 4,5), we have also validated the formation of $\mathrm{NH}_{3}$ as a product of the reaction by using $\mathrm{pH}$ paper (Figure S4a). In this regard, the gas released from the autoclave was tested four times and always turned deep blue, indicating base $\mathrm{NH}_{3}$ as the product of the reaction. $\mathrm{NH}_{3}$ was formed by deprotonation of pyridine in ortho-position with $\mathrm{NH}_{2}^{-}$anions (see main text: Figure 6). Control experiments with pure pyridine $(\mathrm{pH}=8.5)^{2}$ and similar solvothermal conditions do neither result in any overpressure of the autoclaves nor any coloring of $\mathrm{pH}$ paper (Figure S4b). 

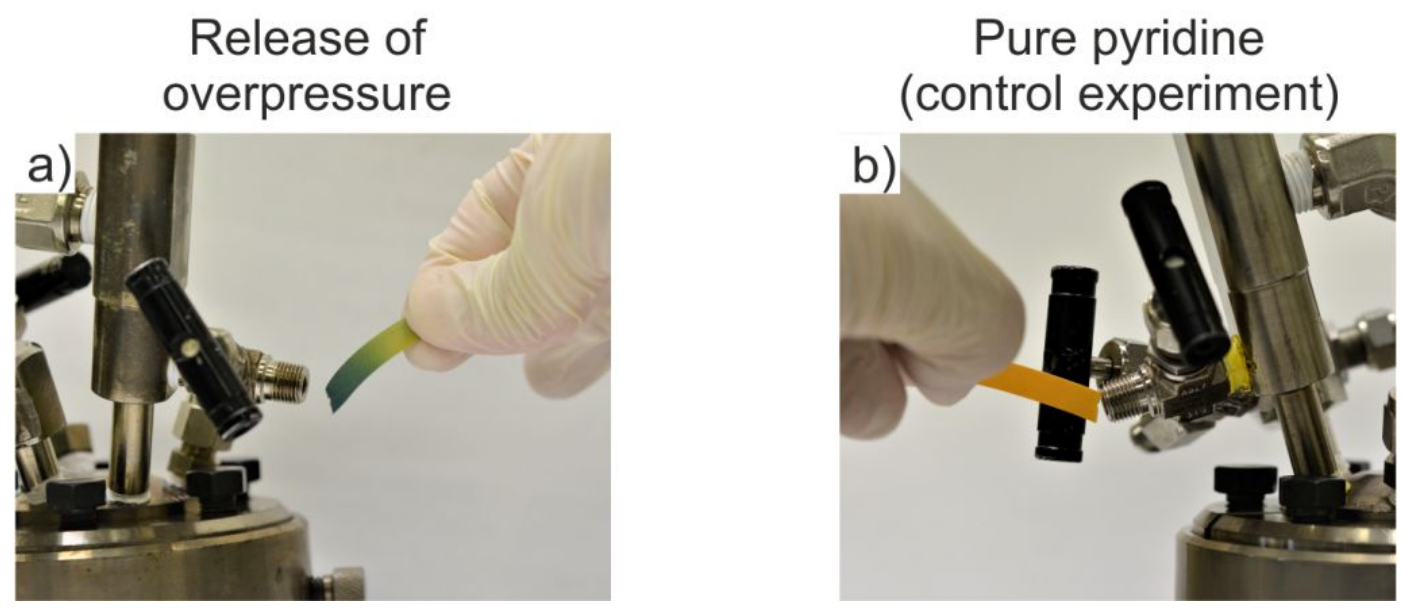

Figure S4. Gas atmosphere of autoclaves after the reaction: a) Release of $\mathrm{NH}_{3}$ as base gas after the reaction of $\mathrm{ZnCl}_{2}$ and $\mathrm{KNH}_{2}$ in pyridine, indicated by $\mathrm{pH}$ indicator paper turning deep blue; b) Reference experiment with pure pyridine.
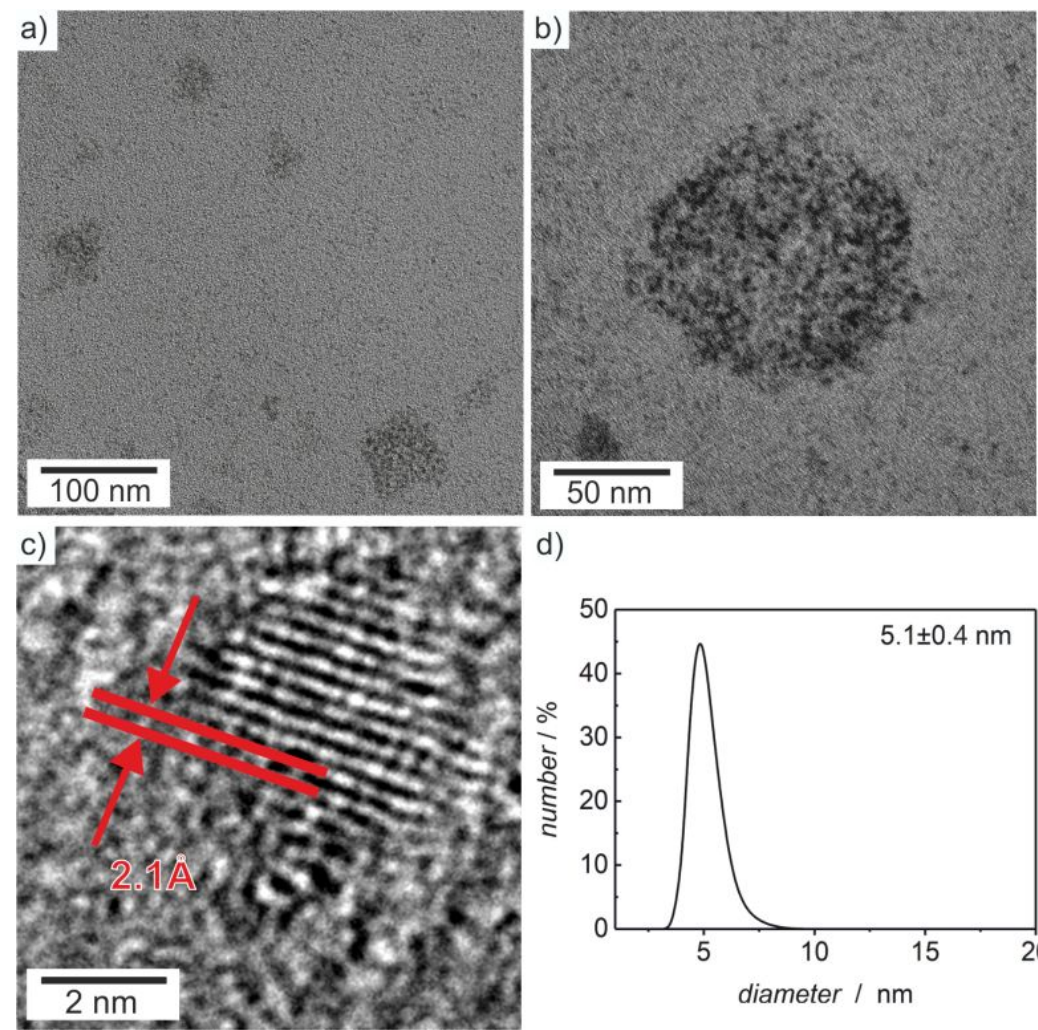

d)

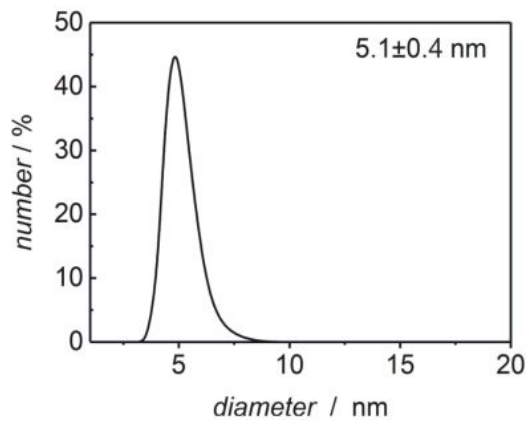

Figure S5. Characterization of $\mathrm{Zn}^{0}$ nanoparticles: $\mathrm{a}+\mathrm{b}$ ) TEM overview images (at different magnification); c) HRTEM image with lattice fringes; d) Particle size distribution (according to statistical evaluation of $>100$ particles). 


\section{4. $\mathrm{Zn}^{0}$ Nanoparticles (One-pot Approach)}

In addition to the two-step $l q-\mathrm{NH}_{3}$ synthesis of $\mathrm{Zn}^{0}$ nanoparticles (see main text: Figures 1,2$), \mathrm{Zn}^{0}$ nanoparticles were prepared via the one-step approach, similar to $\mathrm{Sn}^{0}, \mathrm{~V}^{0}$ and $\mathrm{Mn}^{0}$ (see main text: Figures 7-11). Both two-step $l q-\mathrm{NH}_{3}$ synthesis and one-step approach result in very similar properties. According to TEM overview images, very uniform and spherical $\mathrm{Zn}^{0}$ nanoparticles were obtained (Figure S5a,b). A statistical evaluation based on $>100$ particles results in a mean diameter of $5.1 \pm 0.7 \mathrm{~nm}$ (Figure $\mathrm{S} 5 \mathrm{~d}$ ). Lattice fringes on HRTEM detail images indicate the crystallinity of the as-prepared nanoparticles. The observed lattice distances of $2.1 \AA$ is compatible with bulk- $\mathrm{Zn}^{0} \quad\left(d_{101}\right.$ with $\left.2.09 \AA\right)$ (Figure $\mathrm{S} 5 \mathrm{c}$ ). ${ }^{\mathrm{S} 3}$ The crystallinity and purity of the as-prepared $\mathrm{Zn}^{0}$ nanoparticles was further confirmed by XRD patterns with a large number of nanoparticles. Again the observed Bragg peaks are well in accordance with bulk- $\mathrm{Zn}^{0}$ as a reference (ICDD-No. 00-004-0831; Figure S6).

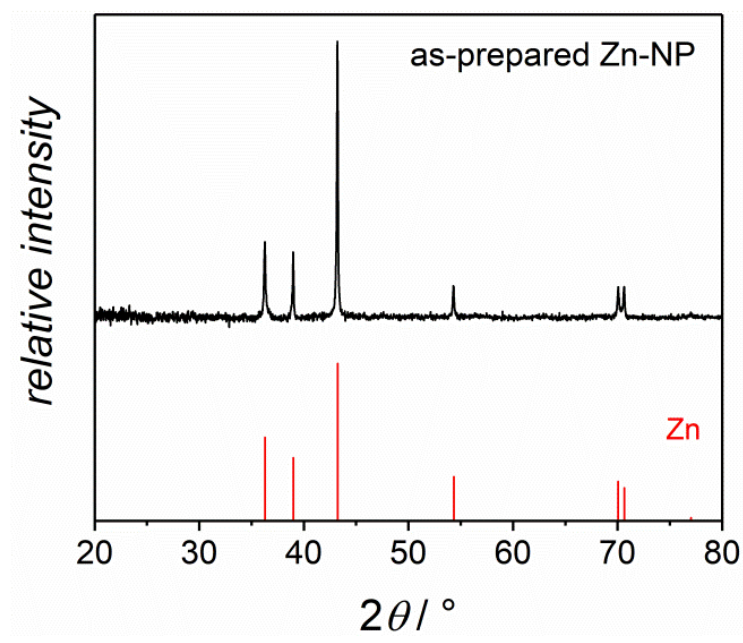

Figure S6. XRD of the as-prepared $\mathrm{Zn}^{0}$ nanoparticles (after pestling with amorphous silica spheres, filling in glass capillaries and sealed under argon; bulk-Znº: ICDD-No. 00-004-0831 as a reference).

\section{Elemental Analysis}

Elemental analysis (EA) of the as-prepared metal nanoparticles was performed to prove their purity (Table S1). Accordingly, all metal nanoparticles - independent of the specific metal - show very comparable contents of carbon, hydrogen and nitrogen, which can be ascribed to the surface functionalization with pyridine. Its total amount of about $10 \mathrm{wt}-\%$ is in accordance with the small size of the metal particles $(5-6 \mathrm{~nm})$. If the weight percent of 
elements were calculated for pyridine in the presence of $90.9 \mathrm{wt} \%$ remain (i.e. the average remain of the metal nanoparticles), the obtained values are in good accordance with the experimental values, which confirms pyridine as surface functionalization on the metal nanoparticles (Table S1). Finally, the similar nitrogen-to-metal contents for all as-prepared metal nanoparticles $(\mathrm{N}: \mathrm{Zn}=0.16, \mathrm{~N}: \mathrm{Sn}=0.20, \mathrm{~N}: \mathrm{V}=0.10, \mathrm{~N}: \mathrm{Mn}=0.07)$ reveal the absence of amide intermediates also in the case of $\mathrm{V}^{0}$ and $\mathrm{Mn}^{0}$, which do not show Bragg peaks in XRD patterns (see main text: Figure 11).

Table S1. Chemical composition of the as-prepared $\mathrm{Zn}^{0}, \mathrm{Sn}^{0}, \mathrm{~V}^{0}$, and $\mathrm{Mn}^{0}$ nanoparticles according to EA.

\begin{tabular}{c|c|c|c|c}
\hline Metal & $\begin{array}{c}\mathbf{C} \\
(\mathbf{w t -} \%)\end{array}$ & $\begin{array}{c}\mathbf{H} \\
\mathbf{( w t - \% )}\end{array}$ & $\begin{array}{c}\mathbf{N} \\
(\mathbf{w t}-\%)\end{array}$ & $\begin{array}{c}\text { Remain (Metal) } \\
(\mathbf{w t - \%})\end{array}$ \\
\hline $\mathbf{Z n}^{\mathbf{0}}$ & 6.6 & 0.9 & 3.1 & 89.4 \\
\hline $\mathbf{S n}^{\mathbf{0}}$ & 5.9 & 0.6 & 2.2 & 91.3 \\
\hline $\mathbf{V}^{\mathbf{0}}$ & 6.3 & 0.7 & 2.5 & 90.5 \\
\hline $\mathbf{M n}^{\mathbf{0}}$ & 5.6 & 0.5 & 1.7 & 92.2 \\
\hline $\begin{array}{c}\text { Pyridine } \\
\text { (reference) }\end{array}$ & $(0.76)$ & $(0.06)$ & $(0.18)$ & $(0.00)^{a}$ \\
\hline
\end{tabular}

${ }^{a}$ Weight percent of elements in the case of pure pyridine.

${ }^{b}$ Weight percent of elements in the case of pyridine in the presence of $90.9 \mathrm{wt}-\%$ remain, which is the average remain of the above metal nanoparticles.

\section{References}

[S1] Merritt, L. L.; Schroeder, E. The crystal structure of 2,2'-bipyridine. Acta Crystallogr. 1956, 9, 801-805.

[S2] Lide, D. R. CRC Handbook of Chemistry and Physics. CRC Press, Boca Raton 2010.

[S3] Nuss, J.; Wedig, U.; Kirfel, A.; Jansen, M. The Structural Anomaly of Zinc: Evolution of Lattice Constants and Parameters of Thermal Motion in the Temperature Range of 40 to 500 K. Z. Anorg. Allg. Chem. 2010, 636, 309-312. 